\title{
Vacancy-type defects in GaN self-assembled nanowires probed using monoenergetic positron beam
}

Cite as: J. Appl. Phys. 125, 175705 (2019); https://doi.org/10.1063/1.5088653

Submitted: 12 January 2019 . Accepted: 16 April 2019 . Published Online: 03 May 2019

Akira Uedono (D), Alexandra-Madalina Siladie (D), Julien Pernot (D), Bruno Daudin (D), and Shoji Ishibashi (iD)
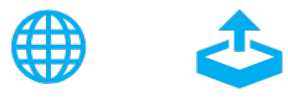

\section{ARTICLES YOU MAY BE INTERESTED IN}

Observation of single optical site of Eu and $\mathrm{Mg}$ codoped $\mathrm{GaN}$ grown by $\mathrm{NH}_{3}$-source molecular beam epitaxy

Journal of Applied Physics 125, 175702 (2019); https://doi.org/10.1063/1.5090893

Polarity conversion of GaN nanowires grown by plasma-assisted molecular beam epitaxy Applied Physics Letters 114, 172101 (2019); https://doi.org/10.1063/1.5094627

Advanced photo-assisted capacitance-voltage characterization of insulator/wide-bandgap semiconductor interface using super-bandgap illumination

Journal of Applied Physics 125, 175704 (2019); https://doi.org/10.1063/1.5089793

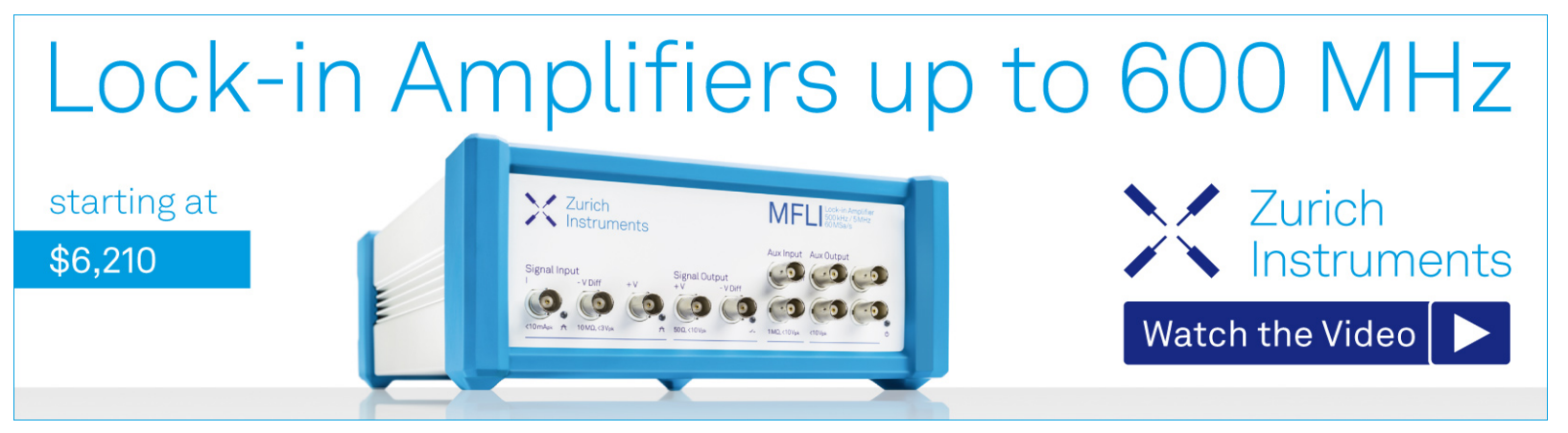




\title{
Vacancy-type defects in GaN self-assembled nanowires probed using monoenergetic positron beam
}

\author{
Cite as: J. Appl. Phys. 125, 175705 (2019); doi: 10.1063/1.5088653 \\ Submitted: 12 January $2019 \cdot$ Accepted: 16 April 2019 . \\ Published Online: 3 May 2019
}

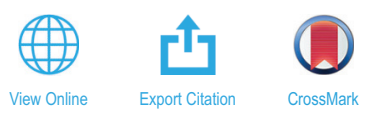

Akira Uedono, $^{1, a)}$ (D) Alexandra-Madalina Siladie, ${ }^{2}$ (D) Julien Pernot, ${ }^{3}$ (D) Bruno Daudin, ${ }^{2}$ (D) and Shoji Ishibashi ${ }^{4}$

\section{AFFILIATIONS}

${ }^{7}$ Division of Applied Physics, Faculty of Pure and Applied Science, University of Tsukuba, Tsukuba, Ibaraki 305-8573, Japan

${ }^{2}$ Université Grenoble Alpes, CEA, INAC-PHELIQS "Nanophysics and Semiconductors" Group, 38000 Grenoble, France

${ }^{3}$ Université Grenoble Alpes, CNRS, Grenoble INP, Institut Néel, 38000 Grenoble, France

${ }^{4}$ Research Center for Computational Design of Advanced Functional Materials (CD-FMat), National Institute of Advanced Industrial Science and Technology (AIST), Tsukuba, Ibaraki 305-8568, Japan

a) uedono.akira.gb@u.tsukuba.ac.jp

\begin{abstract}
Vacancy-type defects in GaN nanowires (NWs) and the trapping of electrons by the vacancies were studied by positron annihilation. Undoped, Si-, and Mg-doped GaN NWs were grown on Si substrates by plasma-assisted molecular beam epitaxy. The major species of vacancies in the undoped and Si-doped samples was identified as a complex between a Ga vacancy and impurities such as oxygen and hydrogen. For the Mg-doped samples, the trapping rate of positrons for such defects decreased with the increase in Mg concentration because of the downward shift of Fermi level position and a resultant shift of the vacancy charge states from neutral (negative) to positive. Under the illumination of a $325-\mathrm{nm}$ He-Cd laser, positrons were found to be trapped by vacancy-type defects, which was attributed to the trapping of excited electrons by these defects.
\end{abstract}

Published under license by AIP Publishing. https://doi.org/10.1063/1.5088653

\section{INTRODUCTION}

Gallium nitride $(\mathrm{GaN})$ and its alloys with $\mathrm{InN}$ and AlN have been used for optoelectronic devices such as light-emitting diodes (LEDs) and ultraviolet lasers over the past decade, and they are now going to play a crucial role in high-frequency and high-power device technology. ${ }^{1}$ The potential of $\mathrm{GaN}$ as a material for electric devices is attributed to its wide bandgap, high thermal conductivity, large breakdown electric field, high saturation electron velocity, etc. Unlike other compound semiconductors such as GaAs or InP, however, GaN-based optical/electrical devices have been mainly fabricated by using GaN grown on non-native substrates such as sapphire, $\mathrm{SiC}$, and $\mathrm{Si}$ because of difficulties in obtaining large-area bulk GaN substrates at low cost. Growth using foreign substrates inevitably causes stress in the GaN layer and introduces dislocations that act as nonradiative recombination centers and degrade the device performance. Tremendous progress has been made on decreasing the dislocation density for planar GaN devices and has resulted in the present success of GaN-based devices. The growth of $\mathrm{GaN}$ nanowires (NWs) has also been extensively studied ${ }^{2,3}$ since they are an attractive option to overcome the issue of the lack of lattice-matched substrates. Indeed, the critical thickness associated with elastic strain relaxation in NW heterostructures has been found to be considerably increased, which is associated with both their reduced diameter and a large surface-to-bulk volume ratio. ${ }^{4}$ Using the molecular beam epitaxy (MBE) technique, GaN-based NWs are grown under nitrogen-rich conditions that promote vertical growth along the $c$-axis direction. ${ }^{5,6}$ It has been reported that GaN NWs can be grown on various substrates, ${ }^{7-10}$ and a broad range of applications that use the NW structure, such as LEDs, lasers, photodetectors, and solar cells, have been demonstrated. ${ }^{11-14}$

Because of the eased elastic strain relaxation in NWs, NW heterostructures are also promising for fabricating $\mathrm{GaN}$ devices with high dopant concentrations and InGaN with a high In content. 
The low efficiency and output power of long wavelength LEDs is assigned to a combination of detrimental factors including poor carrier transport, electron overflow, Auger recombination, and nonradiative recombination. However, the reduction of these effects has been reported in the case of NWs, which is at least partly related to their low density of extended defects and increased dopant solubility limit. ${ }^{15-18}$ The issue of structural defects (dislocation, stacking faults, cracks) and dopant behavior has been widely addressed for GaN NWs and GaN/InGaN NW heterostructures. ${ }^{2,3,19,20}$ By contrast, the properties of point defects are not well known, although it has been suggested that they could drastically affect the external quantum efficiency of $\mathrm{GaN} / \mathrm{InGaN}$ heterostructures. ${ }^{21,22}$ Because vacancy-type defects act as carrier compensators and recombination centers, ${ }^{23}$ knowledge on the behavior of vacancies is crucial to improve the crystal quality and optoelectronic properties of GaN NWs. Positron annihilation is a powerful technique for probing vacancy-type defects in semiconductors. ${ }^{24,25}$ In the present work, we used a monoenergetic positron beam to probe vacancy-type defects in GaN NWs grown on $\mathrm{Si}$ substrates by using the plasma-assisted MBE technique.

\section{EXPERIMENT}

The method for growing GaN NWs by using plasma-assisted MBE is described in Refs. 26-28. A 2-in. Si (111) wafer (n-type, $\leq 5 \mathrm{~m} \Omega \mathrm{cm}$ ) was used as the substrate. Before growing the NWs, substrates were cleaned in a $10 \%$ HF solution and annealed at $870^{\circ} \mathrm{C}$ for $30 \mathrm{~min}$. This preprocess led to the formation of a $7 \times 7$ reconstruction of the $\mathrm{Si}$ surface. During the growth of the NWs, the substrate temperature was kept at $800^{\circ} \mathrm{C}$. Plasma cell radio frequency power was set to $600 \mathrm{~W}$ with a $\mathrm{N}_{2}$ flux of 2.5 standard cubic centimeters per minute ( $\mathrm{sccm}$ ), with a $\mathrm{Ga} /$ active $\mathrm{N}$ nominal flux ratio of 0.3 . P-type doping and n-type doping were obtained by exposing the samples to $\mathrm{Mg}$ or $\mathrm{Si}$ flux from standard effusion cells, respectively. The $\mathrm{Mg}$ cell temperatures were 220 (Mg1), 240 (Mg2), $260(\mathrm{Mg} 3)$, and $280^{\circ} \mathrm{C}(\mathrm{Mg} 4)$, and the Si cell temperatures were at $900(\mathrm{Si} 1), 920(\mathrm{Si} 2)$, and $945^{\circ} \mathrm{C}(\mathrm{Si} 3)$, where the letters in brackets will be used to show the result for each sample in Figs. 4 and 5. The letter " $U$ " is used to describe an undoped sample. An increase in the $\mathrm{Mg}$ or $\mathrm{Si}$ cell temperature, $T_{\text {cell }}$, increases the doping impurity concentration in GaN NWs. The growth time was $7 \mathrm{~h}$. Top- and side-view morphology variations were observed by using a field-emission scanning electron microscope (FESEM) (ZEISS Ultra 55 microscope). Figure 1 shows (a) side and (b) top views of undoped GaN NWs. From the SEM pictures, the length and diameter of the NWs were estimated to be $1-2 \mu \mathrm{m}$ and about $200 \mathrm{~nm}$, respectively. A similar morphology was obtained for the Mg- and Si-doped GaN NWs. Using the top view of the SEM pictures for samples $\mathrm{U}, \mathrm{Mg} 1, \mathrm{Mg} 2, \mathrm{Mg} 3, \mathrm{Mg} 4, \mathrm{Si} 1, \mathrm{Si}$, and Si3, the surface covering rates of the GaN NWs were determined to be $0.71,0.93,0.98,0.87,0.96,0.89,0.80$, and 0.77 , respectively. After the growth, the samples were annealed at $800{ }^{\circ} \mathrm{C}$ in a $\mathrm{N}_{2}$ atmosphere for $30 \mathrm{~min}$.

Details on the positron annihilation technique are described elsewhere. ${ }^{24,25,29}$ The Doppler broadening spectra of the annihilation radiation were measured as a function of the incident positron energy $E$ in darkness and under the illumination of a

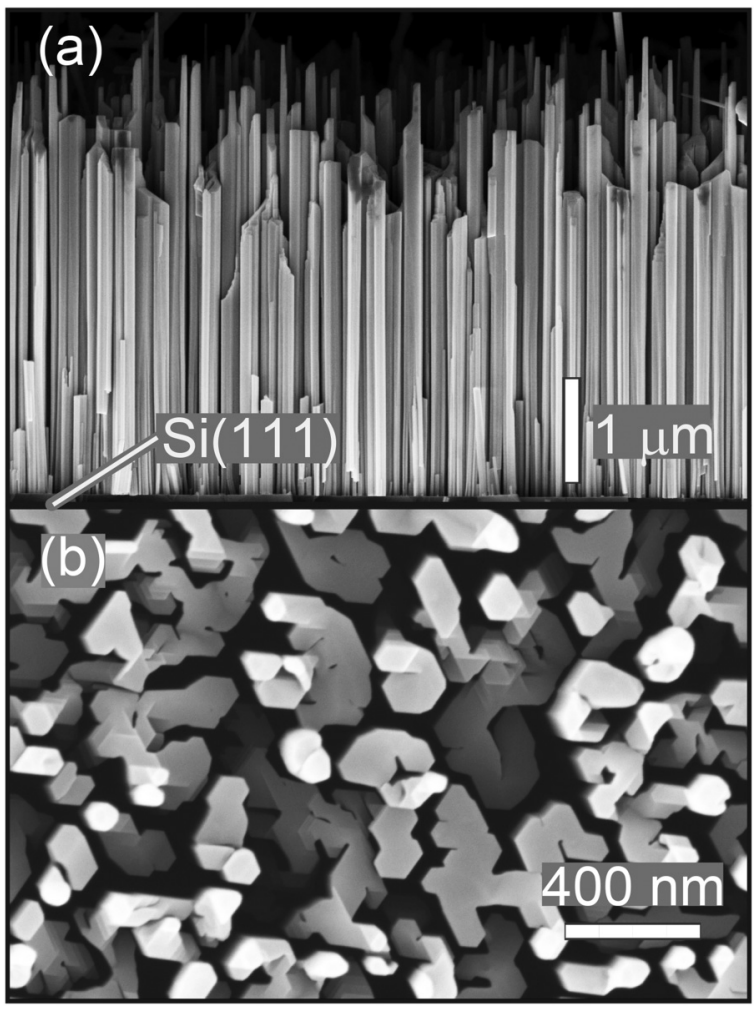

FIG. 1. (a) Side and (b) top views of an undoped sample grown on a Si substrate by using plasma assisted MBE. Length and diameter of NWs were $1-2 \mu \mathrm{m}$ and about $200 \mathrm{~nm}$, respectively.

325-nm He-Cd laser. The total area of the sample was illuminated with an irradiance of about $10 \mathrm{~mW} / \mathrm{cm}^{2}$. The Doppler broadening spectra were characterized by the $S$ parameter, defined as the fraction of annihilation events over the energy range of $510.24-511.76 \mathrm{keV}$, and by the $W$ parameter, defined as the fraction of annihilation events in the ranges of 506.44$508.72 \mathrm{keV}$ and $513.28-515.56 \mathrm{keV}$. They were also measured by using a coincidence technique. ${ }^{24}$ The Doppler broadening spectra for the delocalized state and the trapped states in typical vacancies and vacancy impurity complexes (oxygen and hydrogen) were calculated by using QMAS (Quantum MAterials Simulator) code, and details on the calculation procedure are described elsewhere. ${ }^{30,31}$

\section{RESULTS AND DISCUSSION}

Figure 2 shows the $S$ values of the GaN NWs grown on the $\mathrm{Si}$ substrates as a function of incident positron energy $E$. For the $\mathrm{Mg}$-doped samples, the $\mathrm{Mg}$ cell temperatures are shown in the figure. For the undoped sample, the $S-E$ curves were measured in darkness and under illumination. The result for $c$-face GaN grown by using hydride vapor phase epitaxy (HVPE) is also shown. The mean implantation depth of positrons for $\mathrm{GaN}$ is shown on the 


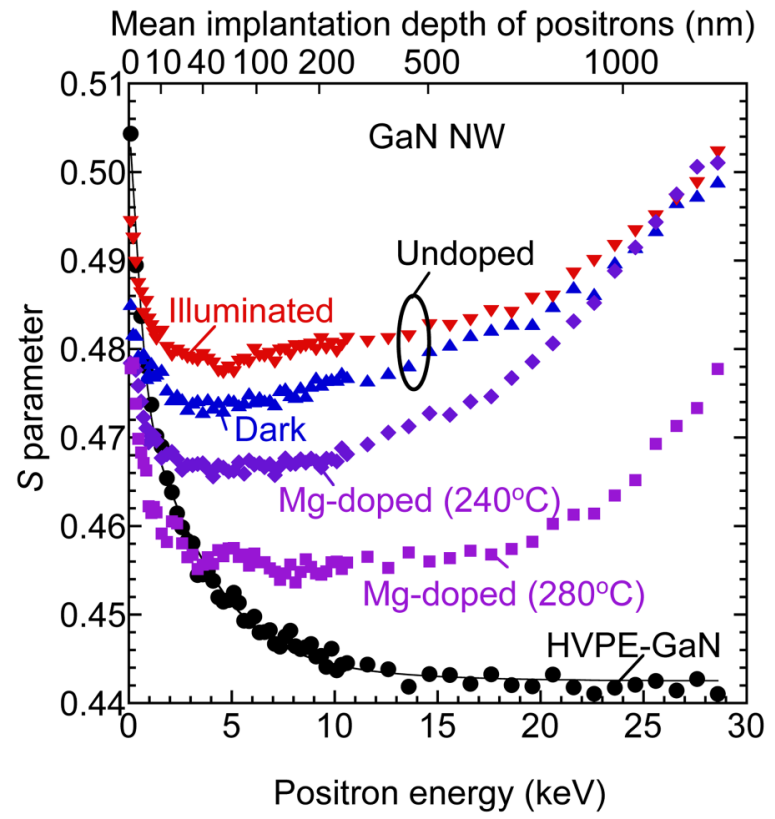

FIG. 2. $S$ parameters as a function of incident positron energy $E$ for an undoped sample measured in darkness and under illumination. $S-E$ curves for Mg-doped samples grown with cell temperatures at 240 and $280^{\circ} \mathrm{C}$ and HVPE-GaN measured in darkness are also shown.

upper horizontal axis. For HVPE-GaN, the $S$ value increased as $E$ decreased, which is due to the diffusion of positrons toward the surface. The $S$ value was saturated at $E>15 \mathrm{keV}$, which is attributed to almost all positrons annihilating in the bulk in this energy range. The diffusion length of positrons in the HVPE-GaN was determined to be $52 \pm 2 \mathrm{~nm}$ using a computer program (VEPFIT) developed by van Veen et al., ${ }^{32}$ which corresponds to the typical diffusion length for defect-free GaN. ${ }^{29,33}$ The $S$ value corresponding to the annihilation in the $\mathrm{Si}$ substrate was determined to be $0.5327 \pm 0.0004$.

For the NW samples, positrons are implanted into both the NWs and the Si substrate. For the undoped sample measured in darkness, the $S$ values saturated as $E$ increased up to $2 \mathrm{keV}$, and they were almost constant in the energy range between 2 and $10 \mathrm{keV}$. Above $E=10 \mathrm{keV}$, the $S$ value started to increase as $E$ increased, which can be attributed to the increased annihilation probability of positrons in the $\mathrm{Si}$ substrate. The $S$ values below $E=20 \mathrm{keV}$ were increased by the illumination, but at $E>20 \mathrm{keV}$, they were almost identical to the values measured in darkness. No illumination effect on the $S$ value was observed for the Si substrate. Thus, the observed increase in the $S$ value under illumination suggests the presence of optically active defects in NWs. We will discuss the illumination effect later. For the $\mathrm{Mg}$-doped samples, the $S$ value below $E=20 \mathrm{keV}$ tended to decrease as $T_{\text {cell }}$ increased. On the one hand, such a decrease could indicate a reduction in the number of vacancies for increasing $\mathrm{Mg}$ doping level. On the other hand, the trapping of positrons by vacancy-type defects is suppressed by the downward shift of the Fermi level position because of the change in the vacancy charge states from negative (neutral) to positive. ${ }^{24,33,34}$ Thus, the observed decrease in the $S$ value for the $\mathrm{Mg}$-doped samples can be attributed to the decrease in the trapping probability of positrons due to the change in the Fermi level position, as will be discussed later.

From the relationship between the $S$ and $W$ values $(S-W$ plot), one can discuss the annihilation sites of positrons in the sample. ${ }^{24}$ In the GaN NW structure, positrons are expected to annihilate from two different materials ( $\mathrm{GaN}$ and $\mathrm{Si}$ ) because the surface coverage rate is smaller than 1 . Then, the observed $S$ value, $S_{\text {obs }}$, is given by a weighted average of the characteristic $S$ values for GaN NWs and $\mathrm{Si}\left(S_{\mathrm{GaN}}\right.$ and $\left.S_{\mathrm{Si}}\right)$,

$$
S_{\mathrm{obs}}=S_{\mathrm{GaN}} F_{\mathrm{GaN}}+S_{\mathrm{Si}}\left(1-F_{\mathrm{GaN}}\right)
$$

where $F_{\mathrm{GaN}}$ is the fraction of positrons annihilating from $\mathrm{GaN}$ NWs. The $W$ value is also obtained by the weighted average of the values for GaN NWs and $\mathrm{Si}\left(W_{\mathrm{GaN}}\right.$ and $\left.W_{\mathrm{Si}}\right)$. Thus, in the $S-W$ plot, the observed $(S, W)$ value lies on a line connecting $\left(S_{\mathrm{GaN}}\right.$, $\left.W_{\mathrm{GaN}}\right)$ and $\left(S_{\mathrm{Si}}, W_{\mathrm{Si}}\right)$.

Figure 3 shows the $S-W$ relationships for the samples shown in Fig. 2. The $(S, W)$ values for the HVPE-GaN and the Si substrate are shown as references for defect-free $\mathrm{GaN}$ and $\mathrm{Si}$, respectively. The $(S, W)$ values for ion-implanted $\mathrm{GaN}(\mathrm{I} / \mathrm{I}-\mathrm{GaN})$ at $\mathrm{RT}$ and $500{ }^{\circ} \mathrm{C}$ are also shown. ${ }^{34,35}$ These values represent the characteristic values for a $\mathrm{Ga}$-vacancy coupled with an $\mathrm{N}$-vacancy, $V_{\mathrm{Ga}} V_{\mathrm{N}}$, and $V_{\mathrm{Ga}}\left(V_{\mathrm{N}}\right)_{2}$, respectively. The arrows in the figure show the change in the $(S, W)$ values with the increase in $E$, and the energy range corresponding to each symbol is shown in the figure. For the undoped sample measured in darkness [Fig. 3(a)], the $(S, W)$ values shifted toward the upper left-hand side with the increase in $E$. Above $E=16 \mathrm{keV}$, they started to shift to the lower right-hand side and approached the $(S, W)$ value for Si. These $(S, W)$ values were found on the line that connects the values for the $\mathrm{Si}$ and I/I-GaN(RT), and they did not approach the line that connects the values for the Si and defect-free GaN. This suggests that positrons implanted into undoped GaN NWs mainly annihilate in the trapped state due to vacancy-type defects. Although the $(S, W)$ values at $2-16 \mathrm{keV}$ are close to the value for I/I-GaN (RT), the major defects in the GaN NWs are not $V_{\mathrm{Ga}} V_{\mathrm{N}}$. Because the value for the NW samples is the weighted average of the values for the GaN NWs and $\mathrm{Si}$, the characteristic value for the GaN NWs should locate in the upper left-hand side of the observed $(S, W)$ values.

For the Mg-doped samples [Figs. 3(c) and 3(d)], the $(S, W)$ values tended to approach the line connecting the $(S, W)$ values for the $\mathrm{Si}$ and $\mathrm{GaN}$ with the increase in the $\mathrm{Mg}$ cell temperature, which suggests that the annihilation probability of positrons in the delocalized state increases due to the suppression of the trapping of positrons by vacancy-type defects. For the undoped sample measured under illumination, the $(S, W)$ values in a low $E$ region (2-8 keV) were found on the line that connects the values of the $\mathrm{Si}$ and $\mathrm{GaN}$, but they depart from the line in a high energy region (16-30 keV), suggesting that the effect of the illumination was limited in the subsurface region. 


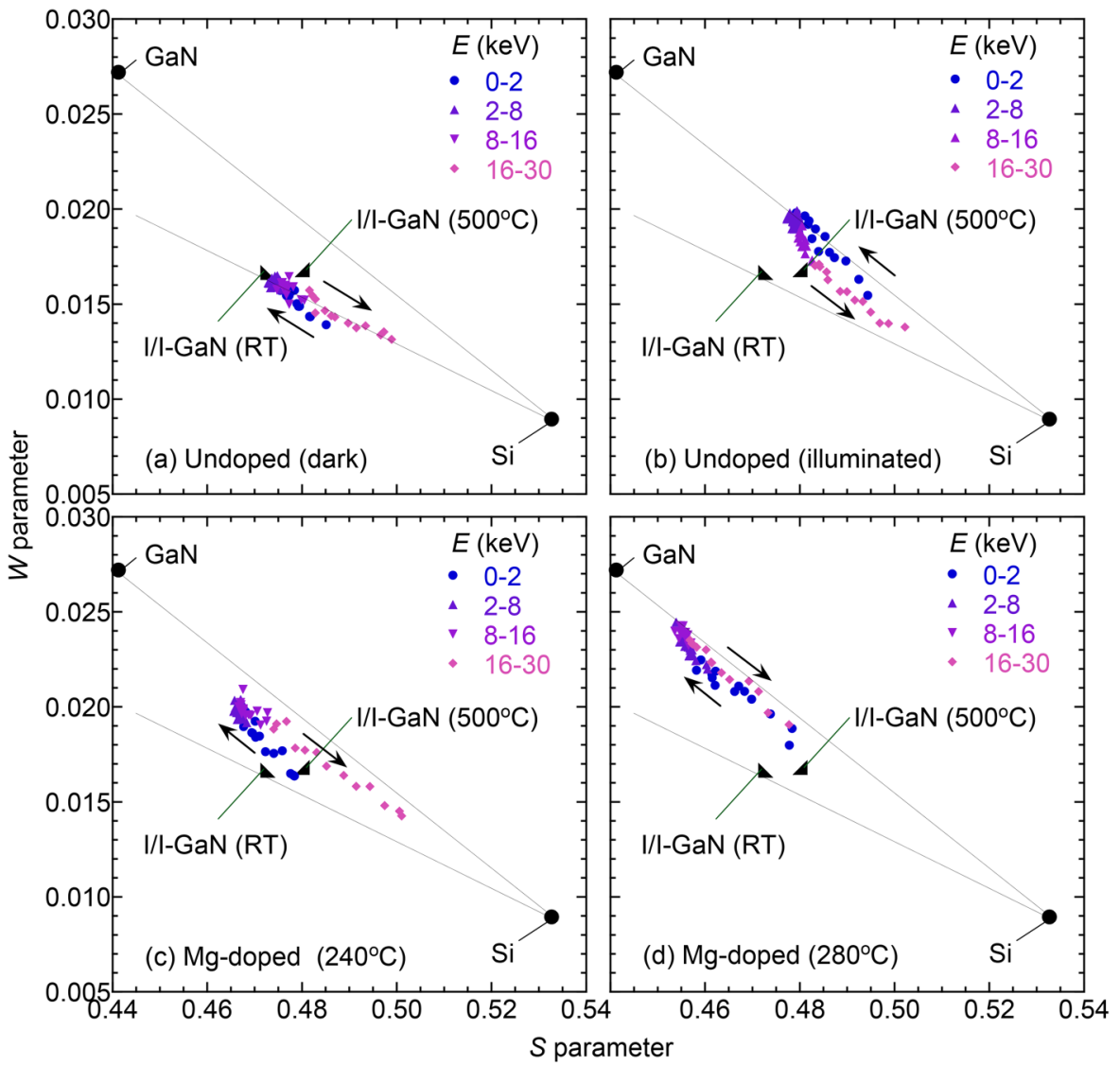

FIG. 3. S-W relationships for an undoped sample measured in darkness (a) and under illumination (b). Results for Mg-doped samples with $T_{\text {cell }}=240^{\circ} \mathrm{C}$ (c) and $280^{\circ} \mathrm{C}$ (d) measured in darkness are also shown. $(S, W)$ values for $\mathrm{Si}, \mathrm{GaN}$, and ion implanted (I/I) GaN with different implantation temperatures (RT and $500^{\circ} \mathrm{C}$ ) are shown as references. ${ }^{34,35}$ The arrows indicate a change in $(S, W)$ value due to an increase in $E$.
Doppler broadening spectra were measured at $E=5.1 \mathrm{keV}$ by using the coincidence technique. The $(S, W)$ values were obtained from those spectra, and the characteristic values for GaN NWs $\left[\left(S_{\mathrm{GaN}}, W_{\mathrm{GaN}}\right)\right]$ were estimated by using Eq. (1), where $F_{\mathrm{GaN}}$ was assumed to be the surface covering rate of the NWs and the $(S, W)$ value for the $\mathrm{Si}$ substrate was used as $\left(S_{\mathrm{Si}}, W_{\mathrm{Si}}\right)$. When positrons are implanted into the NW structure, however, a certain number of positrons are expected to annihilate at the lateral surface of NWs. At $E=5.1 \mathrm{keV}$, the positron annihilation at the top surfaces of NWs is negligible. Thus, the value of $S_{\mathrm{GaN}}$ is given by a weighted average of the characteristic $S$ values for the positron annihilation at the lateral surfaces of NW $\left(S_{\mathrm{GaN}(\mathrm{s})}\right)$ and that inside NWs $\left(S_{\mathrm{GaN}(\mathrm{b})}\right)$,

$$
S_{\mathrm{GaN}}=S_{\mathrm{GaN}(\mathrm{s})} F_{\mathrm{GaN}(\mathrm{s})}+S_{\mathrm{GaN}(\mathrm{b})}\left(1-F_{\mathrm{GaN}(\mathrm{s})}\right),
$$

where $F_{\mathrm{GaN}(\mathrm{s})}$ is the fraction of positrons annihilating at the lateral surfaces of NWs. The value of $W_{\mathrm{GaN}}$ is also obtained by the weighted average of $W_{\mathrm{GaN}(\mathrm{s})}$ and $W_{\mathrm{GaN}(\mathrm{b})}$. When positrons are implanted into solids, they are thermalized and diffuse before the annihilation. If positrons were thermalized near the sidewall of NWs, they could reach the lateral surface. The value of $F_{\mathrm{GaN}(\mathrm{s})}$, therefore, mainly depends on the diffusion length of positrons $\left(L_{\mathrm{d}}\right)$ in NWs.
For undoped and doped samples, the $S-E$ curves in the energy range between 0.1 and $6 \mathrm{keV}$ were analyzed by VEPFIT, and the value of $L_{\mathrm{d}}$ was obtained to be about $5 \mathrm{~nm}$. For the $\mathrm{Mg}$-doped samples, the derived short diffusion length of positrons can be attributed to the band bending at the surface and a resultant introduction of the electric field which suppresses the positron diffusion toward the surface. ${ }^{33}$ Because the $S$ values for the undoped and Si-doped samples were higher than those for the Mg-doped samples, the short diffusion length can be attributed to the trapping of positrons by vacancy-type defects. Assuming that the GaN NWs can be represented by a cylindrical column with a diameter of 200 $\mathrm{nm}$, the ratio of the area within $5 \mathrm{~nm}$ from the lateral surface to the total area was calculated to be $10 \%$, suggesting that about $10 \%$ of positrons implanted into the GaN NWs could reach the lateral surface.

In the present work, the values of $S$ and $W$ corresponding to the positron annihilation at the surface of $m$-face HVPE-GaN were measured. Using these values as $\left(S_{\mathrm{GaN}(\mathrm{s})}, W_{\mathrm{GaN}(\mathrm{s})}\right) \quad(0.4957 \pm$ $0.0008,0.0115 \pm 0.0001)$, the values of $\left(S_{\mathrm{GaN}(\mathrm{b})}, W_{\mathrm{GaN}(\mathrm{b})}\right)$ were estimated. Figure 4 shows the $S_{\mathrm{GaN}(\mathrm{b})}-W_{\mathrm{GaN}(\mathrm{b})}$ relationships for the samples measured (a) in darkness and (b) under illumination, where the statistical error of the $(S, W)$ value is close to the symbol used in the figure. The results for the HVPE-GaN (denoted as "GaN") and the Si substrate, which correspond to the positron 


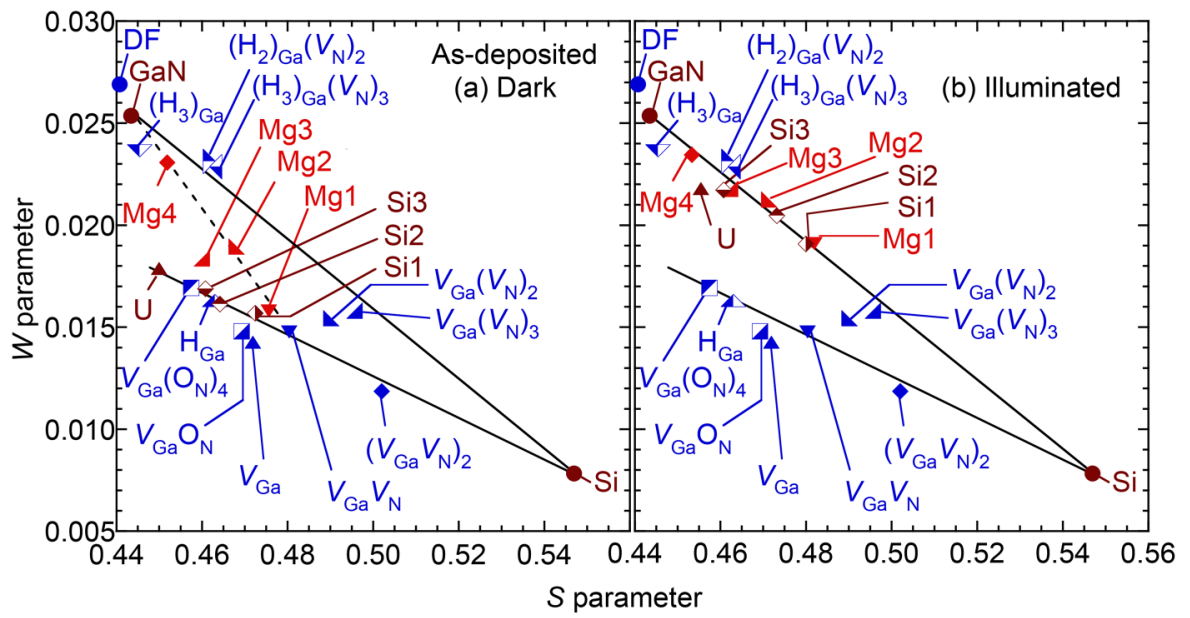

FIG. 4. (S,W values (brown and red) corresponding to annihilation of positrons in GaN NWs for undoped (U), Mg-doped (Mg1, Mg2, Mg3, and Mg4), and Si-doped samples (Si1, Si2, and $\mathrm{Si3}$ ). Measurements were done (a) in darkness and (b) under illumination. $(S, W)$ values for HVPE-GaN (denoted as $\mathrm{GaN}$ ) and $\mathrm{Si}$ are shown as references (brown). In (a), the dotted line connects $(S, W)$ values for $\mathrm{Mg}$-doped samples. ( $S, W$ ) values (blue) were calculated by using the QMAS code ${ }^{30}$ for positron annihilation in defect-free (DF), $V_{\mathrm{Ga}}, V_{\mathrm{Ga}}\left(V_{\mathrm{N}}\right)_{n}, \quad\left(V_{\mathrm{Ga}} V_{\mathrm{N}}\right)_{2}$, and their complexes with oxygen and hydrogen. annihilation in the free states in $\mathrm{GaN}$ and $\mathrm{Si}$, are also shown. The calculated $(S, W)$ values for the annihilation of positrons in the delocalized state (defect-free: DF), vacancy complexes between $V_{\mathrm{Ga}} \mathrm{s}$ and $V_{\mathrm{N}} \mathrm{s}\left[\left(V_{\mathrm{Ga}}\right)_{\mathrm{m}}\left(V_{\mathrm{N}}\right)_{\mathrm{n}}\right]$, and vacancy-impurity complexes are also shown in the figures (blue symbols). The calculated $(S, W)$ value for $\mathrm{DF}-\mathrm{GaN}$ lies to the upper left of the values for HVPE-GaN. The difference between the values obtained by experiments and simulations could be due to several causes, such as the limitations of first-principles calculations applied to Doppler broadening spectra, the experimental background, and/or the energy resolutions of $\mathrm{Ge}$ detectors.

Most of the GaN NWs surface consists of m-plane side wall facets, which makes favorable the incorporation of $\mathrm{O}$ impurities. ${ }^{36}$ Moreover, it has been demonstrated that preferential incorporation of $\mathrm{Mg}$ from the side walls is associated with $\mathrm{H}$ incorporation, assessed by the observation of $\mathrm{Mg}-\mathrm{H}$ Raman modes in Mg-doped GaN NWs. ${ }^{20,37}$ Consequently, it will be assumed in the following that major impurities in GaN NWs are oxygen and hydrogen. As shown in Fig. 4, the coupling between $V_{\mathrm{Ga}}$ and oxygen located at nitrogen sites, $\mathrm{O}_{\mathrm{N}} \mathrm{s}$, caused the upper left-hand side shift from the $(S, W)$ value for $V_{\mathrm{Ga}}$, but the values for $V_{\mathrm{Ga}} \mathrm{O}_{\mathrm{N}}$ and $V_{\mathrm{Ga}}\left(\mathrm{O}_{\mathrm{N}}\right)_{4}$ are located on the line that connects the values for the $V_{\mathrm{Ga}} V_{\mathrm{N}}$ and $\mathrm{Si}$ and do not approach the value for the defect-free GaN. Theoretical works on the role of hydrogen in GaN have been reported in Refs. 38 and 39. In the present work, $V_{\mathrm{Ga}}$ containing hydrogen atoms denoted as $\left(\mathrm{H}_{\mathrm{n}}\right)_{\mathrm{Ga}}$, where $\mathrm{n}$ is the number of hydrogen atoms in $V_{\mathrm{Ga}}$. The formation energies of $V_{\mathrm{Ga}}$ and $V_{\mathrm{Ga}} V_{\mathrm{N}}$ are decreased by hydrogenation, but the formation energy of $V_{\mathrm{N}}$ containing a hydrogen atom is higher than "pure" $V_{\mathrm{N}}$. Since the properties of the hydrogenated $V_{\mathrm{Ga}}$ with one or two hydrogen atoms, such as the transition levels in the bandgap, are not so much different from those of $V_{\mathrm{Ga}}$, they act as acceptor-type defects. Because the positron trapping rate for donor-type defects is small, ${ }^{24}$ we mainly consider hydrogenated $V_{\mathrm{Ga}}$ and vacancy clusters in the present work. When hydrogen atoms are located inside $V_{\mathrm{Ga}}$, the $(S, W)$ value tends to approach the value for defect-free GaN with the increase in the number of hydrogen atoms in $V_{\mathrm{Ga}}$. The same is true for vacancy clusters coupled with multiple hydrogen atoms, such as $\left(\mathrm{H}_{2}\right)_{\mathrm{Ga}}\left(V_{\mathrm{N}}\right)_{2}$ and $\left(\mathrm{H}_{3}\right)_{\mathrm{Ga}}\left(V_{\mathrm{N}}\right)_{3}$.

The $(S, W)$ value for the undoped sample (denoted by " $U$ ") is close to the calculated value for $V_{\mathrm{Ga}}\left(\mathrm{O}_{\mathrm{N}}\right)_{4}$. As shown in Fig. 2, the $S$ value decreased quickly as $E(<2 \mathrm{keV})$ increased, suggesting that the diffusion length of positrons in the NWs was short. This means that almost all positrons annihilated from the trapped state in the NWs. Thus, the major defect species of the undoped NWs was identified as vacancy-oxygen complexes such as $V_{\mathrm{Ga}}\left(\mathrm{O}_{\mathrm{N}}\right)_{4}$. For the Si-doped sample, the $(S, W)$ value lies on the line connecting the values for $\mathrm{Si}$ and $V_{\mathrm{Ga}}\left(\mathrm{O}_{\mathrm{N}}\right)_{4}$. As $T_{\text {cell }}$ increased ( $\mathrm{Si} 1 \rightarrow \mathrm{Si} 2 \rightarrow \mathrm{Si} 3$ ), the $(S, W)$ value tended to approach the value for the undoped sample, suggesting that the concentration of $V_{\mathrm{Ga}}\left(\mathrm{O}_{\mathrm{N}}\right)_{4}$ for the sample with a low $\mathrm{Si}$ concentration $[\mathrm{Si}$ ] was lower than that for the undoped sample, and it increased as [Si] increased. A similar phenomenon was reported for Si-doped AlGaN, ${ }^{40}$ which could be due to the recombination between $V_{\mathrm{Ga}}$ and Si. Because the formation energy of $V_{\mathrm{Ga}}$ decreases as the Fermi level position increases, ${ }^{23}$ however, growth with a high $T_{\text {cell }}$ could increase the defect concentration. Since the values for other defects such as $V_{\mathrm{Ga}}, V_{\mathrm{Ga}} V_{\mathrm{N}}$, and $\mathrm{H}_{\mathrm{Ga}}$ are also located on the line that connects the values for $V_{\mathrm{Ga}}\left(\mathrm{O}_{\mathrm{N}}\right)_{4}$ and $\mathrm{Si}$, the possibility of the presence of such defects cannot be neglected. For the $\mathrm{Mg}$-doped sample fabricated with $T_{\text {cell }}=220^{\circ} \mathrm{C}(\mathrm{Mg} 1)$, the $(S, W)$ value is close to the line discussed above, suggesting that the major defects in this sample were not so much different from those of the Si-doped sample fabricated with a low $T_{\text {cell }}$. As $T_{\text {cell }}$ increased, the $(S, W)$ values located on the same line and tended to approach the value for defect-free GaN. This can be attributed to the suppression of the trapping of the defects by the downward shift of the Fermi level position.

For the undoped sample, the $(S, W)$ value was shifted above by illumination [Fig. 4(b)] and approached the line connecting the values for $\mathrm{GaN}$ and $\mathrm{Si}$. As shown in Fig. 2, the $S$ value was increased by the illumination, suggesting an increase in the trapping probability of positrons by vacancy-type defects. Thus, the behavior of the $(S, W)$ value was not due to the increase in the annihilation probability in the free state in GaN. For planar GaN grown on the $\mathrm{Si}$ substrate and ion implanted $\mathrm{GaN}$, an increase in the 


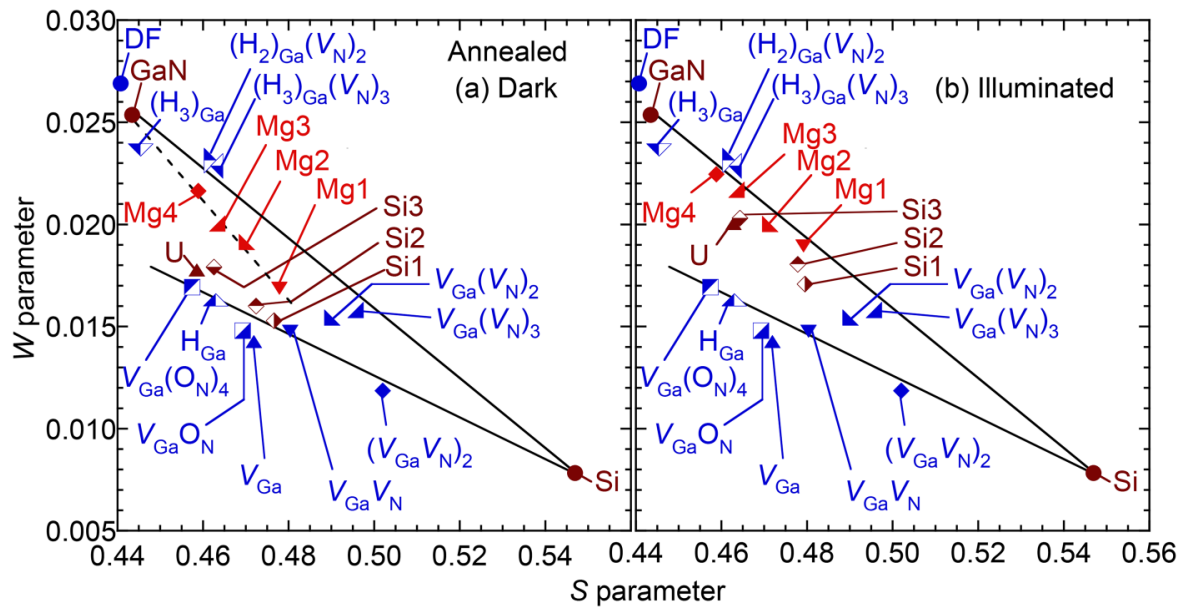

FIG. 5. ( $S, W$ values (brown and red) corresponding to annihilation of positrons in GaN NWs for undoped, Mg-doped, and Si-doped samples after $800^{\circ} \mathrm{C}$ annealing. Measurements were done (a) in darkness and (b) under illumination.
$S$ value under illumination was reported. ${ }^{34,41,42}$ The transition of the charge state of vacancy-type defects $(V)$ from positive to neutral (or neutral to negative), $V^{+} \rightarrow V^{0}$ (or $V^{0} \rightarrow V^{-}$), increases the trapping probability of positrons because positrons are attracted by vacancies through the Coulomb force. ${ }^{24}$ Thus, the observed change in the $(S, W)$ value can be attributed to the capture of photon-excited electrons by vacancy-type defects in the GaN NWs. Because the $(S, W)$ value for the undoped sample under illumination is close to the values for vacancy clusters containing hydrogen atoms, such as $\left(\mathrm{H}_{2}\right)_{\mathrm{Ga}}\left(V_{\mathrm{N}}\right)_{2}$ and $\left(\mathrm{H}_{3}\right)_{\mathrm{Ga}}\left(V_{\mathrm{N}}\right)_{3}$, these defects are possible candidates for optically active defects observed in the present case. For the Si-doped and $\mathrm{Mg}$-doped samples under illumination, because a similar trend was observed, a similar type of defect complexes is likely to be the major trapping center of positrons.

Figure 5 shows the $S_{\mathrm{GaN}(\mathrm{b})}-W_{\mathrm{GaN}(\mathrm{b})}$ plots for the samples after annealing at $800^{\circ} \mathrm{C}$. Compared with the results before annealing, the $(S, W)$ values tended to shift toward the lower right. For ion implanted $\mathrm{GaN}$, the major defect species in an as-implanted sample was $V_{\mathrm{Ga}} V_{\mathrm{N}} \cdot{ }^{34}$ After annealing at $1000^{\circ} \mathrm{C}$, the clustering of the vacancy-type defects occurred, and larger vacancy clusters such as $\left(V_{\mathrm{Ga}} V_{\mathrm{N}}\right)_{3}$ were introduced. Thus, the annealing at $800^{\circ} \mathrm{C}$ was unlikely to anneal out the defects in the GaN NWs but to promote vacancy clustering. Because the $(S, W)$ values for vacancy clusters, such as $\left(V_{\mathrm{Ga}} V_{\mathrm{N}}\right)_{2}, V_{\mathrm{Ga}}\left(V_{\mathrm{N}}\right)_{2}$, and $V_{\mathrm{Ga}}\left(V_{\mathrm{N}}\right)_{3}$, located in the lower right in the $S-W$ plot, the introduction of such defects could be the origin of the shift of the $(S, W)$ values obtained after the annealing.

\section{SUMMARY}

We used positron annihilation spectroscopy to study vacancytype defects in GaN NWs grown on Si by MBE. For an undoped sample, the major defect species was identified as a $V_{\mathrm{Ga}}$ coupled with impurities such as oxygen and hydrogen. A similar trend was observed for Si-doped samples. For Mg-doped samples, the positron trapping rate for vacancy-type defects was suppressed because of the downward shift of the Fermi level position due to the doping. For samples under illumination, the effect of the annihilation of positrons trapped by vacancy-type defects was observed.
This was attributed to the shift of the defect charge state due to the trapping of electrons excited by illumination. After annealing at $800^{\circ} \mathrm{C}$, the clustering of vacancy-type defects was observed. We have shown that positron annihilation parameters are sensitive to vacancy-type defects in GaN NWs and that positron annihilation spectroscopy is a useful tool for optimizing the growth condition of the nanowire structure.

\section{ACKNOWLEDGMENTS}

This work was supported by JSPS KAKENHI (Grant No. 16H06424). A part of this work was also supported by MEXT "Program for research and development of next-generation semiconductor to realize energy-saving society" and the French Agence Nationale pour la Recherche (Project No. ANR-15-CE24-0006).

\section{REFERENCES}

${ }^{1}$ H. Amano et al., J. Phys. D Appl. Phys. 51, 163001 (2018).

${ }^{2}$ S. Zhao, H. P. T. Nguyen, M. G. Kibria, and Z. Mi, Prog. Quant. Electron. 44, 14 (2015).

${ }^{3}$ C. Zhao, N. Alfaraj, R. C. Subedi, J. W. Liang, A. A. Alatawi, A. A. Alhamoud, M. Ebaid, M. S. Alias, T. K. Ng, and B. S. Ooi, Prog. Quant. Electron. 61, 1 (2018).

${ }^{\mathbf{4}}$ F. Glas, Phys. Rev. B 74, 121302(R) (2006).

${ }^{\mathbf{5}}$ M. Yoshizawa, A. Kikuchi, M. Mori, N. Fujita, and K. Kishino, Jpn. J. Appl. Phys. 36, L459 (1997).

${ }^{6}$ M. A. Sanchez-Garcia, E. Calleja, E. Monroy, F. J. Sanchez, F. Calle, E. Munoz, and R. Beresford, J. Cryst. Growth 183, 23 (1998).

${ }^{7}$ M. Tchernycheva, C. Sartel, G. Cirlin, L. Travers, G. Patriarche, J.-C. Harmand, L. S. Dang, J. Renard, B. Gayral, L. Nevo, and F. Julien, Nanotechnology 18, 385306 (2007).

${ }^{8}$ F. Furtmayr, M. Vielemeyer, M. Stutzmann, J. Arbiol, S. Estradé, F. Peiro, J. R. Morante, and M. Eickhoff, J. Appl. Phys. 104, 034309 (2008).

${ }^{9}$ V. Consonni, M. Hanke, M. Knelangen, L. Geelhaar, A. Trampert, and H. Riechert, Phys. Rev. B 83, 035310 (2011).

${ }^{10}$ S. Zhao, M. G. Kibria, Q. Wang, H. P. T. Nguyen, and Z. Mi, Nanoscale 5, 5283 (2013).

${ }^{11}$ H.-M. Kim, Y.-H. Cho, H. Lee, S. I. Kim, S. R. Ryu, D. Y. Kim, T. W. Kang, and K. S. Chung, Nano Lett. 4, 1059 (2004). 
${ }^{12}$ A. Kikuchi, M. Kawai, M. Tada, and K. Kishino, Jpn. J. Appl. Phys. 43, L1524 (2004).

${ }^{13}$ S. Gradecak, F. Qian, Y. Li, H.-G. Park, and C. M. Lieber, Appl. Phys. Lett. 87, 173111 (2005).

${ }^{14}$ H. Sekiguchi, K. Kishino, and A. Kikuchi, Electron. Lett. 44, 151 (2008).

${ }^{15} \mathrm{~J}$. Xie, X. Ni, Q. Fan, R. Shimada, Ü Özgür, and H. Morkoç, Appl. Phys. Lett. 93, 121107 (2008)

${ }^{16}$ H. Pham, T. Nguyen, K. Cui, S. Zhang, M. Djavid, A. Korinek, G. A. Botton, and Z. Mi, Nano Lett. 12, 1317 (2012).

${ }^{17}$ H. Pham, T. Nguyen, M. Djavid, K. Cui, and Z. Mi, Nanotechnology 23, 194012 (2012).

${ }^{18}$ Z. Fang, E. Robin, E. Rozas-Jiménez, A. Cros, F. Donatini, N. Mollard, J. Pernot, and B. Daudin, Nano Lett. 15, 6794 (2015).

${ }^{19}$ G. Tourbot, C. Bougerol, A. Grenier, M. D. Hertog, D. Sam-Giao, D. Cooper, P. Gilet, B. Gayral, and B. Daudin, Nanotechnology 22, 075601 (2011).

${ }^{\mathbf{2 0}}$ A.-M. Siladie, L. Amichi, N. Mollard, I. Mouton, B. Bonef, C. Bougerol, A. Grenier, E. Robin, P.-H. Jouneau, N. Garro, A. Cros, and B. Daudin, Nanotechnology 29, 255706 (2018).

${ }^{21}$ C. Haller, J.-F. Carlin, G. Jacopin, D. Martin, R. Butté, and N. Grandjean, Appl. Phys. Lett. 111, 262101 (2017).

${ }^{22}$ Y. Tu, Y. Ruan, L. Zhu, Q. Tu, H. Wang, J. Chen, Y. Lu, Y. Gao, T.-M. Shih, Z. Chen, and Y. Lin, J. Appl. Phys. 123, 161544 (2018).

${ }^{23}$ C. G. Van de Walle and J. Neugebauer, J. Appl. Phys. 95, 3851 (2004).

${ }^{24} \mathrm{R}$. Krause-Rehberg and H. S. Leipner, Positron Annihilation in Semiconductors, Solid-State Sciences (Springer-Verlag, Berlin, 1999), p. 127.

${ }^{25} \mathrm{~F}$. Tuomisto and I. Makkonen, Rev. Mod. Phys. 85, 1583 (2013).

${ }^{\mathbf{2 6}}$ R. Songmuang, O. Landré, and B. Daudin, Appl. Phys. Lett. 91, 251902 (2007).

${ }^{27}$ O. Landré, R. Songmuang, J. Renard, E. Bellet-Amalric, H. Renevier, and B. Daudin, Appl. Phys. Lett. 93, 183109 (2008).
${ }^{28}$ R. Mata, K. Hestroffer, J. Budagosky, A. Cros, C. Bougerol, H. Renevier, and B. Daudin, J. Cryst. Growth 334, 177 (2011).

${ }^{29}$ A. Uedono, S. Ishibashi, N. Oshima, and R. Suzuki, Jpn. J. Appl. Phys. 52 08JJ02 (2013).

${ }^{30}$ S. Ishibashi and A. Uedono, J. Phys. Conf. 505, 012010 (2014).

${ }^{31}$ S. Ishibashi, T. Tamura, S. Tanaka, M. Kohyama, and K. Terakura, Phys. Rev. B 76, 153310 (2007).

${ }^{32}$ A. van Veen, H. Schut, M. Clement, J. M. M. de Nijs, A. Kruseman, and M. R. IJpma, Appl. Surf. Sci. 85, 216 (1995).

${ }^{33}$ A. Uedono, S. Ishibashi, K. Tenjinbayashi, T. Tsutsui, K. Nakahara, D. Takamizu, and S. F. Chichibu, J. Appl. Phys. 111, 014508 (2012).

${ }^{34}$ A. Uedono, S. Takashima, M. Edo, K. Ueno, H. Matsuyama, W. Egger, T. Koschine, C. Hugenschmidt, M. Dickmann, K. Kojima, S. F. Chichibu, and S. Ishibashi, Phys. Status Solidi B 255, 1700521 (2018).

${ }^{35}$ A. Uedono, S. Takashima, M. Edo, K. Ueno, H. Matsuyama, H. Kudo, H. Naramoto, and S. Ishibashi, Phys. Status Solidi B 252, 2794 (2015).

${ }^{36}$ S. R. Xu, Y. Hao, J. C. Zhang, Y. R. Cao, X. W. Zhou, L. A. Yang, X. X. Ou, K. Chen, and W. Mao, J. Cryst. Growth 312, 3521 (2010).

${ }^{37}$ J. E. Northrup, Phys. Rev. B 77, 045313 (2008).

${ }^{38}$ Y. S. Puzyrev, T. Roy, M. Beck, B. R. Tuttle, R. D. Schrimpf, D. M. Fleetwood, and S. T. Pantelides, J. Appl. Phys. 109, 034501 (2011).

${ }^{39}$ J. L. Lyons, A. Alkauskas, A. Janotti, and C. G. Van de Walle, Phys. Status Solidi B 252, 900 (2015).

${ }^{40}$ A. Uedono, K. Tenjinbayashi, T. Tsutsui, Y. Shimahara, H. Miyake, K. Hiramatsu, N. Oshima, R. Suzuki, and S. Ishibashi, J. Appl. Phys. 111, 013512 (2012).

${ }^{41}$ A. Uedono, T. Fujishima, Y. Cao, Y. Zhang, N. Yoshihara, S. Ishibashi, M. Sumiya, O. Laboutin, W. Johnson, and T. Palacios, Appl. Phys. Lett. 104, 082110 (2014).

${ }^{42}$ A. Uedono, T. Tanaka, N. Ito, K. Nakahara, W. Egger, C. Hugenschmidt, S. Ishibashi, and M. Sumiya, Thin Solid Films 639, 78 (2017). 